(617) 871-7820; Fax: (617) 871-7049; E-mail: david.glass@novartis.com.

1. McKinsey, T.A., Zhang, C.L., and Olson, E.N. 2002. Signaling chromatin to make muscle. Curr. Opin. Cell Biol. 14:763-772.

2. Suzuki, N., et al. 2007. NO production results in suspension-induced muscle atrophy through dislocation of neuronal NOS. J. Clin. Invest. 117:2468-2476. doi:10.1172/JCI30654.

3. McKinsey, T.A., Zhang, C.L., Lu, J., and Olson, E.N 2000. Signal-dependent nuclear export of a histone deacetylase regulates muscle differentiation. Nature. 408:106-111.

4. Liu, Y., Randall, W.R., and Schneider, M.F. 2005 Activity-dependent and -independent nuclear fluxes of HDAC4 mediated by different kinases in adult skeletal muscle. J. Cell Biol. 168:887-897.

5. Kirkin, V., and Dikic, I. 2007. Role of ubiquitin- and Ubl-binding proteins in cell signaling. Curr. Opin Cell Biol. 19:199-205.

6. Zhao, X., Sternsdorf, T., Bolger, T.A., Evans, R.M. and Yao, T.-P. 2005. Regulation of MEF2 by histone deacetylase 4- and SIRT1 deacetylase-mediated lysine modifications. Mol. Cell. Biol. 25:8456-8464.

7. Gregoire, S., and Yang, X.-J. 2005. Association with class IIa histone deacetylases upregulates the sumoylation of MEF2 transcription factors. $\mathrm{Mol}$.
Cell. Biol. 25:2273-2287.

8. Hanson, P., et al. 1997. Acute corticosteroid myopathy in intensive care patients. Muscle Nerve. 20:1371-1380.

9. Glass, D.J. 2005. A signaling role for dystrophin: inhibiting skeletal muscle atrophy pathways. Cancer Cell. 8:351-352.

10. Acharyya, S., et al. 2005. Dystrophin glycoprotein complex dysfunction: a regulatory link between muscular dystrophy and cancer cachexia. Cancer Cell. 8:421-432.

11. Glass, D.J. 2005. Skeletal muscle hypertrophy and atrophy signaling pathways. Int. J. Biochem. Cell Biol. 37:1974-1984

12. Sandri, M., et al. 2004. Foxo transcription factors induce the atrophy-related ubiquitin ligase atrogin-1 and cause skeletal muscle atrophy. Cell. 117:399-412.

13. Stitt, T.N., et al. 2004. The IGF-1/PI3K/Akt pathway prevents expression of muscle atrophy-induced ubiquitin ligases by inhibiting FOXO transcription factors. Mol. Cell. 14:395-403.

14. Kamei, Y., et al. 2004. Skeletal muscle FOXO1 (FKHR) transgenic mice have less skeletal muscle mass, down-regulated Type I (slow twitch/red muscle) fiber genes, and impaired glycemic control. J. Biol. Chem. 279:41114-41123.

15. Bodine, S.C., et al. 2001. Identification of ubiquitin ligases required for skeletal muscle atrophy. Science. 294:1704-1708.

16. Potthoff, M.J., et al. 2007. Histone deacetylase degradation and MEF2 activation promote the formation of slow-twitch myofibers. J. Clin. Invest. 117:2459-2467. doi:10.1172/JCI31960.

17. Grozdanovic, Z., Gosztonyi, G., and Gossrau, R. 1996. Nitric oxide synthase I (NOS-I) is deficient in the sarcolemma of striated muscle fibers in patients with Duchenne muscular dystrophy, suggesting an association with dystrophin. Acta Histochem. 98:61-69.

18. Crosbie, R.H., Barresi, R., and Campbell, K.P. 2002. Loss of sarcolemma nNOS in sarcoglycan-deficient muscle. FASEB J. 16:1786-1791.

19. Wehling, M., Spencer, M.J., and Tidball, J.G. 2001. A nitric oxide synthase transgene ameliorates muscular dystrophy in $\mathrm{mdx}$ mice. J. Cell Biol. 155:123-132.

20. Archer, J.D., Vargas, C.C., and Anderson, J.E. 2006. Persistent and improved functional gain in $\mathrm{mdx}$ dystrophic mice after treatment with L-arginine and deflazacort. FASEB J. 20:738-740.

21. Yasukawa, T., et al. 2005. S-nitrosylation-dependent inactivation of Akt/protein kinase B in insulin resistance. J. Biol. Chem. 280:7511-7518.

22. Anderson, I. 2006. Deacetylase inhibitors aid recovery in muscular dystrophy. Lancet Neurol. 5:906

\title{
Rheostat regulation of integrin-mediated leukocyte adhesion
}

\author{
Ivor S. Douglas ${ }^{1}$ and Themistocles Dassopoulos ${ }^{2}$
}

1Division of Pulmonary Sciences and Critical Care Medicine, University of Colorado at Denver and Health Sciences Center, and Denver Health Medical Center, Denver, Colorado, USA. ${ }^{2}$ Meyerhoff Inflammatory Bowel Disease Center, Johns Hopkins University Medical Center, Baltimore, Maryland, USA.

\begin{abstract}
The homing of activated $\mathrm{T}$ lymphocytes to the gut in inflammatory bowel diseases is dependent on their coordinated, integrin-mediated adhesion and de-adhesion to substrates and blood vessel walls. In this issue of the JCI, Park and colleagues reveal a key modulatory role of a binding site within $\beta$ integrins, known as the ADMIDAS domain, in controlling integrin de-adhesion in mice (see the related article beginning on page 2526). These observations add to our growing understanding of how integrin adhesiveness is regulated and raise the notion of the existence of a biological rheostat for lymphocyte homing. Disturbed migratory rheostat tone could account for variations in interindividual immune responses observed in patients with inflammatory bowel disease or other lymphocyte-mediated inflammatory disorders. These findings will inform future strategies to design small molecules for the treatment of a spectrum of chronic inflammatory conditions.
\end{abstract}

Multiple lines of evidence from animal models and diseased humans have

Nonstandard abbreviations used: ADMIDAS, adjacent to metal ion-dependent adhesion site; CAM, cell adhesion molecule; $\mathrm{CD}$, Crohn disease; $\mathrm{HEV}$, high endothelial venule; IBD, inflammatory bowel disease; ILK-1, integrin-linked kinase-1; MAdCAM-1, mucosal addressin cell adhesion molecule-1; UC, ulcerative colitis.

Conflict of interest: The authors have declared that no conflict of interest exists.

Citation for this article: J. Clin. Invest. 117:2391-2395 (2007). doi:10.1172/JCI33376. defined the central role of gut-homing effector and regulatory $\mathrm{T}$ lymphocytes in the pathogenesis of inflammatory bowel diseases (IBDs) (1).

Integrins are cell surface-expressed, heterodimeric glycoproteins that play a prominent role in diverse immune cell interactions as well as in regulating lymphocyte migration, homing, survival, and proliferation $(2,3)$. The addressin receptors are expressed on gut lamina propria postcapillary venules and on high endothelial venules (HEVs; specialized postcapillary endothelial structures in mesenteric lymph nodes and Peyer's patches) and function as organ-specific molecular "zip codes" for lymphocytes by facilitating integrin-mediated lymphocyte homing and transendothelial migration in a classical 4-step process. After initial tethering and rolling (step 1), lymphocytes are activated (step 2) and then undergo an activation-dependent change in conformational state of the $\alpha$ and $\beta$ integrins, resulting in the upregulation of integrin adhesiveness (step 3). Ligand-receptor avidity and affinity interactions between lymphocyte integrins and addressin receptors are regulated by inside-out signaling in response to cytoskeletal stress, G proteincoupled receptor activation, or receptor tyrosine kinase receptor activation (3). This highaffinity binding is essential for lymphocyte migration across the endothelium (step 4) and into target organs (4).

\section{Integrin-mediated adhesion and} de-adhesion in leukocyte migration Transendothelial lymphocyte diapedesis into target tissues such as the gut is 


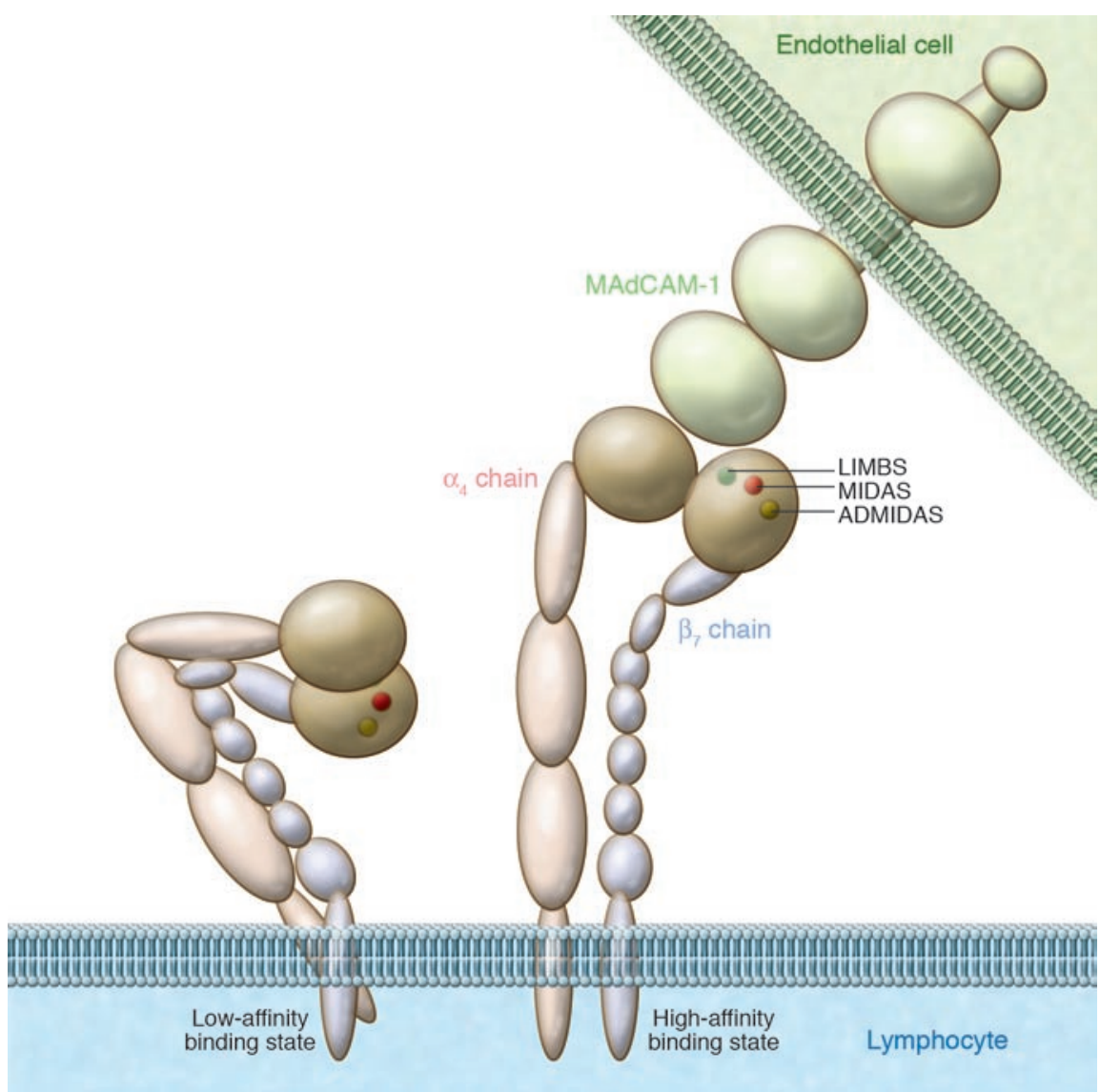

regulated both by integrin-receptor adhesion and by de-adhesion. This tightly regulated shuffling of leading and trailing edges of transendothelial migratory cells is mediated largely by cytoskeletal plasticity signaled by the integrin receptors in response to microenvironment matrix proteins (such as fibronectin via the extracellular RGD domains) and integrin receptors of the immunoglobulin superfamily. This process of sticking and unsticking facilitates remarkably efficient migration along chemokine gradients. In this issue of the JCI, Park and coworkers (5) present, in meticulous detail, the effects on lymphocyte de-adhesion and migration of a targeted activating mutation of one of three conformational regulatory extracellular domains of the integrin $\beta$ subunit, a ligand-binding regulatory domain known as the adjacent to metal ion-dependent adhesion site (ADMIDAS; Figure 1). Multiple cation-binding sites are found in both $\alpha$ and $\beta$ integrin subunits. The ADMIDAS domain regulates conformational activation by binding cations $\left(\mathrm{Ca}^{2+}, \mathrm{Mg}^{2+}\right.$, and $\left.\mathrm{Mn}^{2+}\right)$, thereby allosterically stabilizing alternative con- formations of the integrin and affecting cellular adherence and release during adhesion and migration. The authors demonstrate, in genetically engineered mice carrying a targeted, activating mutation of the ADMIDAS domain, that lymphocyte migration is paralyzed both in vivo and in vitro because of impaired de-adhesion and excessive lymphocyte adhesiveness during migratory shuffling through HEVs in the gut. The impairment in de-adhesion was affected by fewer cell surface-expressed integrin molecules than in wild-type control animals, suggesting a particularly powerful regulatory role for ADMIDAS-mediated conformational activation in regulating lymphocyte de-adhesion.

\section{Role of integrin-dependent T lymphocyte migration in IBDs}

Integrin $\alpha_{4} \beta_{7}$ is the major lymphocyteexpressed ligand for addressins in the postcapillary venules of the intestinal lamina propria and in the HEVs of the gut secondary lymphoid organs, such as Peyer's patches and mesenteric lymph nodes (Figure 2). Integrin $\alpha_{4} \beta_{7}$ is also a

\section{Figure 1}

The interaction of the $3 \beta$-chain cation-binding sites of lymphocyte $\alpha_{4} \beta_{7}$ integrin in the unliganded state and in the liganded conformation with endothelial cell MAdCAM-1. Insideout signaling coordinates cation-dependent allosteric conformational activation of the $\alpha_{4} \beta_{7}$ integrin with the transition from a low-affinity binding state to an "open-hinge," high-affinity binding state. This facilitates MAdCAM-1 binding and firm adhesion to $\alpha_{4} \beta_{7}$. The orientation in the MAdCAM-1-liganded form of the linear cluster of 3 metal-binding sites (ligand-induced metal-binding site [LIMBS], metal ion-dependent adhesion site [MIDAS], and ADMIDAS) in the ligand-binding head region of the integrin $\beta$ subunit von Willebrand factor type $\mathrm{A}$ ( $\beta \mathrm{A}$ or I-like) domain are shown in green, red, and yellow, respectively. ADMIDAS and ligand-induced metal-binding site are regulators of adhesion and de-adhesion and contribute to adhesive rheo-tone. As reported by Park et al. (5) in this issue of the $\mathrm{JCl}$, ADMIDAS regulates de-adhesion of the lymphocyte's trailing edge integrins, thus facilitating transendothelial migration along chemokine gradients.

well-characterized gut-homing molecule and is important for the transendothelial migration of activated T cells in IBDs (6). Expression of mucosal addressin cell adhesion molecule-1 (MAdCAM-1), a cognate ligand for $\alpha_{4} \beta_{7}$, is increased in the inflamed mucosa of patients with ulcerative colitis (UC) and Crohn disease (CD) $(7,8)$ (Figure 3 ). The alternative $\alpha$ chain partner for $\beta_{7}$ is $\alpha_{E}$, also known as CD103. $\alpha_{E} \beta_{7}$ is expressed on CD25 ${ }^{+}$Tregs, is a potential MAdCAM-1 ligand, and is implicated in lymphocyte binding to intestinal epithelial cells (9).

Expression of endothelial cell adhesion molecules (CAMs) such as MAdCAM-1 is upregulated in several lymphocyte-dependent models of IBD $(10,11)$. The Cotton-top tamarin (CTT) is a New World primate that experiences a spontaneous, chronic colitis marked by periodic relapses of acute inflammation. This colitis model closely mimics human UC in its histologic features and response to pharmacologic agents. In landmark studies, administration of anti- $\alpha_{4}$ and anti- $\alpha_{4} \beta_{7}$ monoclonal antibodies attenuated acute and chronic colitis in the CTT model 
A

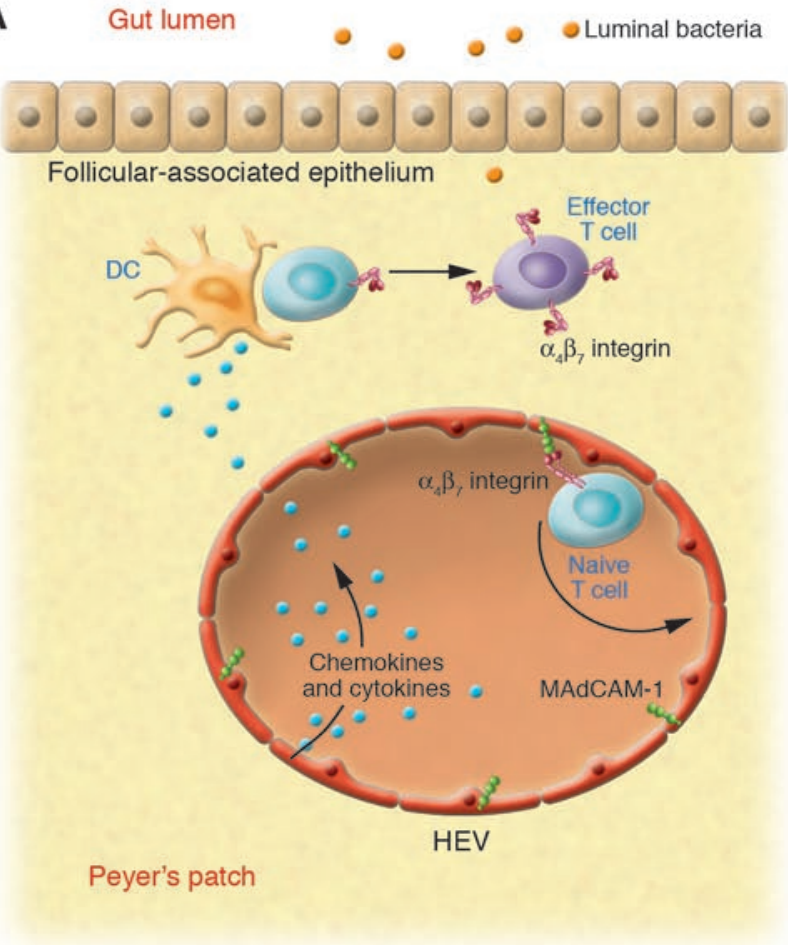

B Gut lumen

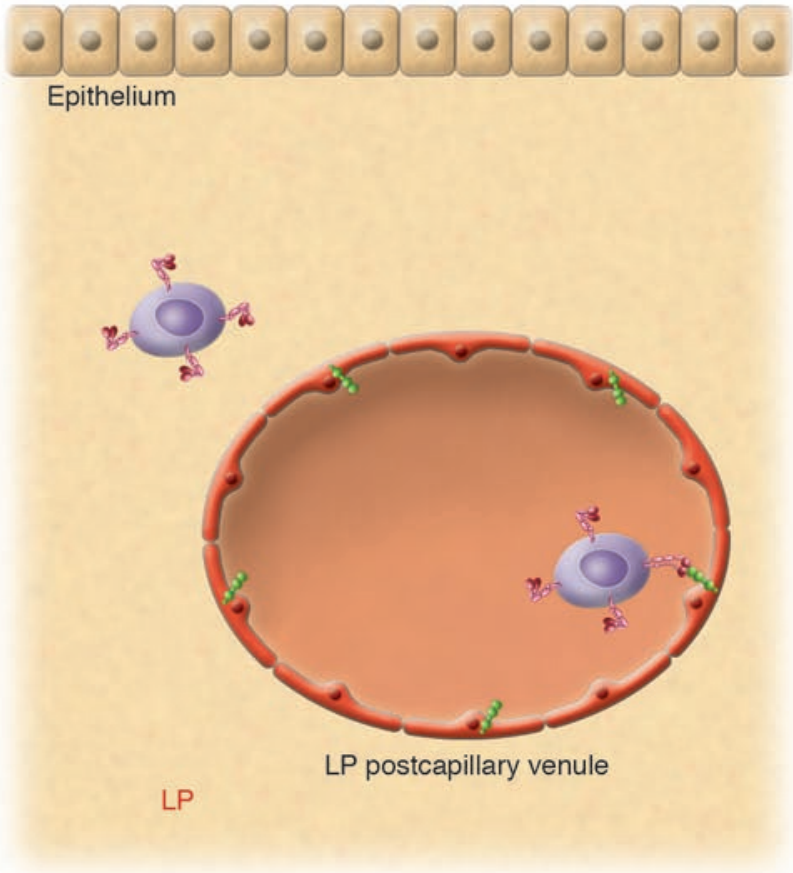

Figure 2

Gut-targeted homing of $\alpha_{4} \beta_{7}{ }^{+}$naive and effector T cells. The gut-associated lymphoid tissues consist of $(\mathbf{A})$ inductive sites, including secondary lymphoid organs, organized lymphoid tissues such as Peyer's patches, and mesenteric lymph nodes that are responsible for the inductive phase of the immune response; and (B) effector sites, including epithelium and lamina propria (LP) of the intestinal mucosa. Endothelial cells of HEVs secrete chemokines that attract naive T cells. Endothelial chemokines trigger the activation of $\alpha_{4} \beta_{7}$ integrin, which binds MAdCAM-1 on HEVs with high affinity, leading to the firm adhesion of rolling lymphocytes. The $T$ cells then transmigrate across the endothelium into the T cell zone of the secondary lymphoid organs, where dendritic cells present antigen to the T cells. Antigen sampling in Peyer's patches is controlled by a layer of specialized epithelial cells known as the follicular-associated epithelium. Peyer's patch dendritic cells imprint guthoming specificity on T cells (34), which become activated and upregulate integrin $\alpha_{4} \beta_{7}$ expression. These activated T cells then migrate to the epithelium and lamina propria of the gut (B).

$(12,13)$. Treatment with anti-MAdCAM-1 antibodies ameliorates intestinal inflammation in several IBD models, including dextran sodium sulfate-induced colitis $(14,15)$ and the ileitis of SAMP1/Yit mice (16). Antisense MAdCAM-1 oligonucleotides suppress the development of trinitrobenzene sulfonate-induced colitis in mice, a $\mathrm{T}$ cell-mediated animal model of IBD (17). Neutralizing monoclonal antibodies directed against other endothelial CAMs have also shown efficacy in some animal models by decreasing inflammation and mucosal damage (18-20). CAMs have also been demonstrated to be of importance in non-IBD models of bowel inflammation, such as graft-versus-host disease (21).

In humans with CD and UC, therapeutic targeting of gut integrins and addressins may represent a treatment option, particularly for patients with refractory disease. Natalizumab, a humanized monoclonal antibody against $\alpha_{4}$ integrin, is efficacious in inducing remission in patients with moderate to severe CD and elevated levels of C-reactive protein (22). However, therapeutic integrin modulation for the treatment of autoimmune diseases may have adverse effects on adaptive immune responses to infection: patients with multiple sclerosis and CD that were treated with natalizumab (23) developed progressive multifocal leukoencephalopathy, possibly as a result of inhibition of lymphocyte trafficking and impaired immune surveillance, presumably leading to reactivation of a clinically latent JC polyomavirus infection. MLN02, an anti- $\alpha_{4} \beta_{7}$ humanized monoclonal antibody, has shown promise in reducing gut inflammation without the attendant risk of more widespread immunosuppression in UC (24).

The $\mathrm{CD} 4{ }^{+} \mathrm{CD} 45 \mathrm{RB}^{\text {high }}$ adoptive transfer model of inflammatory colitis has provided important insights into the pathogenesis of gut inflammation. SCID or $\mathrm{Rag}^{-/-}$mice and athymic rats reconstituted with $\mathrm{CD} 4{ }^{+} \mathrm{CD} 45 \mathrm{RB}^{\text {high }} \mathrm{T}$ lymphocytes from normal animals develop a Th1-type chronic colitis (25). As in human IBD, mucosal microflora are critically important in this model: the immunodeficient mice do not develop colitis in a germ-free environment, and antibiotic therapy ameliorates the inflammation. The power of this model lies in the demonstration that cotransfer of CD45RB low (i.e., mature) $\mathrm{T}$ lymphocytes prevents colitis. Elegant work has demonstrated that this cell population contains Tregs that antagonize the proinflammatory effector $T$ cells via production of antiinflammatory cytokines such as IL-10 and TGF- $\beta$ $(26,27)$. The findings of Park et al. (5) do not indicate whether the striking paralysis of lymphocyte migration in ADMIDAS mutants results in functional impairment of Tregs as well as effector $\mathrm{T}$ cells. This 
A

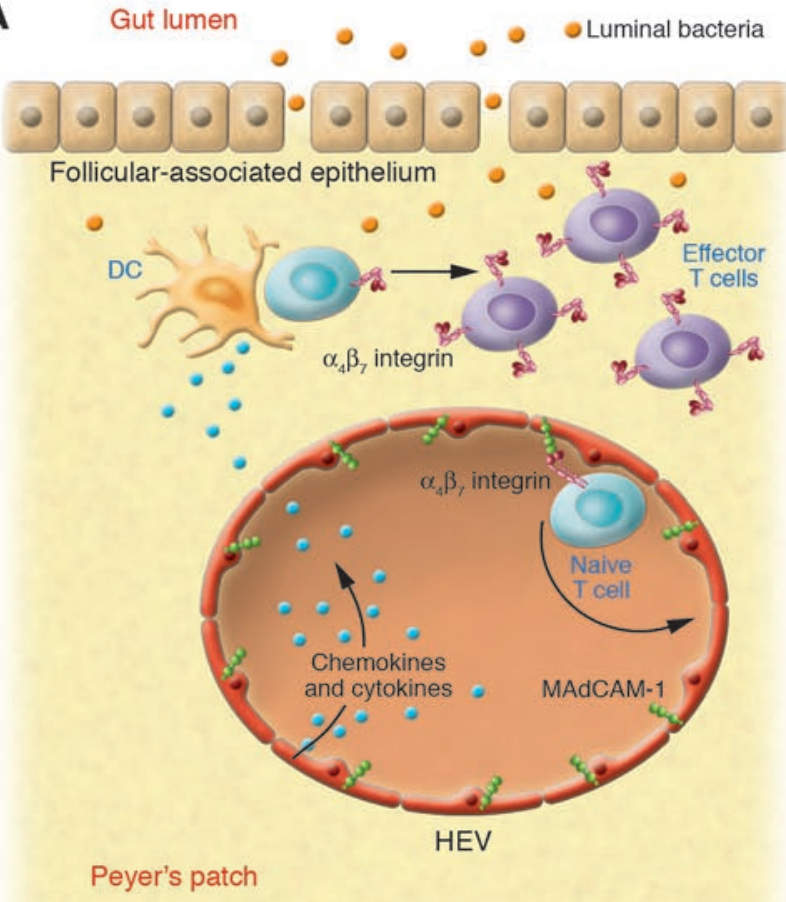

B Gut lumen

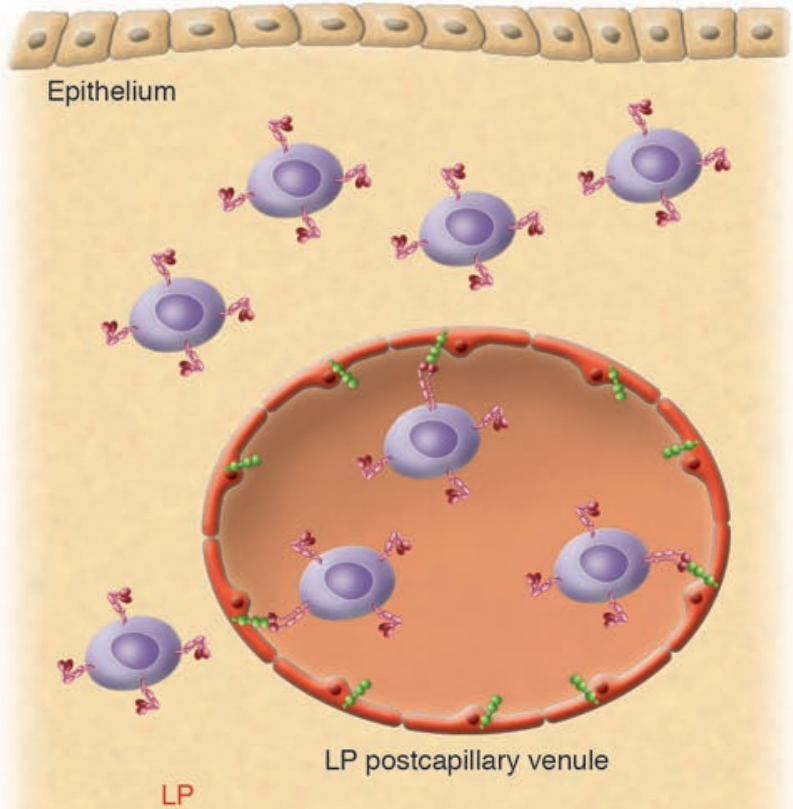

Figure 3

Activated $\alpha_{4} \beta_{7} 7^{+}$T cells drive gut inflammation in IBD. (A) Barrier dysfunction of the follicular-associated epithelium may represent a crucial step in the initiation of gut inflammation. The sites of initial inflammation in IBD are the lymphoid follicles, in which the aphthoid lesions originate from small erosions of the follicular-associated epithelium $(35,36)$. In IBD, the expression of proinflammatory cytokines (e.g., TNF- $\alpha$, IFN- $\gamma$, and IL-12) leads to endothelial cell activation and upregulation of the expression of MAdCAM-1 and other endothelial CAMs. (B) Greater numbers of activated gut-homing T cells migrate through the endothelium to the lamina propria and the epithelium, where they are involved in tissue injury.

is of potential importance because Stassen et al. (28) have reported that activated human $\mathrm{CD}_{25} 5^{+} \alpha_{4} \beta_{7}{ }^{+}$Tregs induce, through direct cell-cell contact, differentiation of a discrete IL-10-producing subset of T suppressor (Tr1-like) cells. It remains uncertain whether this integrindependent Treg effect is relevant to disease or a response to specific $\alpha_{4} \beta_{7}$ inhibition, because $\beta_{7}^{-/-}$Tregs were as efficient as wild-type cells in suppressing inflammation in the murine adoptive transfer colitis model (29). Further, Annacker and colleagues have demonstrated that $\alpha_{\mathrm{E}}{ }^{+}$ dendritic cells, but not $\alpha_{\mathrm{E}} \beta_{7}{ }^{+}$Tregs, are essential for development of lymphocytemediated colitis in the adoptive transfer model (30).

The effect of the constitutively activating ADMIDAS mutation (23) was abrogation of the magnitude and extent of inflammation in a $\mathrm{T}$ cell-transfer colitis model using Rag-1/-/- mice, in association with dramatically impaired gut accumulation of activated $\mathrm{T}$ lymphocytes. The observation of extraintestinal seques- tration in the bone marrow or spleen of ADMIDAS mutant lymphocytes might explain, in part, the reduced gut homing observed in this model. Using intravital microscopy studies, the authors demonstrate circumstantially that this is not the case, because ADMIDAS mutant lymphocyte trafficking through Peyer's patches is preserved and there are stable circulating lymphocyte numbers present as detected by competitive homing assays. The results of these studies strongly support the notion that the ADMIDAS domain is necessary for the de-adhesive phase of lymphocyte migration and is a potentially druggable target for novel therapeutic intervention.

\section{Rheostatic adhesive tone may regulate leukocyte homing and migration to target organs}

The observations of Park et al. (5) raise several questions about the relevance of integrin-mediated adhesive/de-adhesive balance to human disease both in the gut and in other target organs affected by lymphocyte- and autoimmune-mediated inflammation, including bronchiolitis, interstitial pneumonitis, systemic sclerosis, rheumatoid disease, autoimmune hepatitis, cholangitis, autoimmune endocrinopathies, and neurological disorders.

The genetically engineered, constitutively active ADMIDAS domain phenotype (5) represents an extreme form of impaired de-adhesion during lymphocyte migration. It is possible that naturally occurring conformational variants of integrin ADMIDAS, whether regulated by SNPs or by epigenetic or posttranslational modifications, could contribute to a spectrum of lymphocyte "migratory tone" that contributes to both the risk for autoimmune disease and/or the magnitude of host adaptive immune responses to environmental stimuli. The interplay of integrin adhesive/de-adhesive resistance could function as a biological rheostat for target organ homing by activated effector and/or regulatory lymphocytes. In future studies it will be important to address whether constitutively active ADMIDAS 
domain-containing Tregs are also compromised. Conceptually, this hypothesis might be extended to explain variations in interindividual responses to intraluminal gut flora or inhaled environmental antigens. Individuals with high de-adhesive rheo-tone may be at a lower risk for developing diseases such as IBDs, asthma, or interstitial pneumonia.

Supportive of the integrin-addressin rheostat concept are associations between an ICAM-1/CD54 (K469E) SNP and the occurrence, disease localization, and severity of human IBD (31-33). Migratory rheostat resistance could be affected both in the lymph node and in the target organ in response to environmental stimuli such as matrix interactions and integrin coregulatory molecules (e.g., integrin-linked kinase-1; ILK-1). ILK-1 is a serine/threonine kinase that binds the cytoplasmic tail of $\beta$ integrins and through actin cytoskeletal modifications is involved in coordinating intercellular focal adhesions. Mice carrying a targeted $\mathrm{T}$ cell-specific deletion of ILK- 1 have a $50 \%$ reduction in chemokine-mediated $\mathrm{T}$ cell chemotaxis without altered static adhesion to VCAM-1 or changes in cell surface expression of $\beta$ integrins (24). It is unknown whether this is a result of altered de-adhesion, but this may be a further regulator of the leukocyte migratory rheostat.

The migratory rheostat concept could inform future strategies for personalized diagnosis and therapeutic small-molecule screening and design in a spectrum of chronic inflammatory conditions including IBD, interstitial and granulomatous lung diseases, asthma, rheumatoid arthritis, and a wide variety of immune-mediated neurological conditions including multiple sclerosis. Strategies that selectively target homing molecules, avoid systemic immunosuppression, and regulate end-organ inflammation represent exciting opportunities for future therapies for these diseases.

\section{Acknowledgments}

This work was supported in part by NIH grant HL070940 to Ivor S. Douglas.

Address correspondence to: Ivor S. Douglas, Pulmonary and Critical Care Medicine, Denver Health and University of Colorado
Health Sciences Center, Department of Medicine, MC 4000, 777 Bannock Street, Denver, Colorado 80204, USA. Phone: (303) 436-5905; Fax: (303) 436-7249; E-mail: idouglas@dhha.org.

1. Xavier, R.J., and Podolsky, D.K. 2007. Unravelling the pathogenesis of inflammatory bowel disease. Nature. 448:427-434.

2. Hynes, R.O. 2002. Integrins: bidirectional, allosteric signaling machines. Cell. 110:673-687.

3. Kinashi, T. 2005. Intracellular signalling controlling integrin activation in lymphocytes. Nat. Rev. Immunol. 5:546-559.

4. Sackstein, R. 2005. The lymphocyte homing receptors: gatekeepers of the multistep paradigm. Curr. Opin. Hematol. 12:444-450.

5. Park, E.J., et al. 2007. Aberrant activation of integrin $\alpha_{4} \beta_{7}$ suppresses lymphocyte migration to the gut. J. Clin. Invest. 117:2526-2538. doi:10.1172/JCI31570.

6. Salmi, M., and Jalkanen, S. 2005. Lymphocyte homing to the gut: attraction, adhesion, and commitment. Immunol. Rev. 206:100-113.

7. Briskin, M., et al. 1997. Human mucosal addressin cell adhesion molecule- 1 is preferentially expressed in intestinal tract and associated lymphoid tissue. Am. J. Pathol. 151:97-110.

8. Souza, H.S., Elia, C.C., Spencer, J., and MacDonald, T.T. 1999. Expression of lymphocyte-endothelial receptor-ligand pairs, alpha4beta7/MAdCAM-1 and OX40/OX40 ligand in the colon and jejunum of patients with inflammatory bowel disease. Gut. 45:856-863.

9. Oshitani, N., et al. 2003. Differential expression of homing receptor CD103 on lamina propria lymphocytes and association of CD103 with epithelial adhesion molecules in inflammatory bowel disease. Int. J. Mol. Med. 12:715-719.

10. McDonald, S.A., Palmen, M.J., Van Rees, E.P., and MacDonald, T.T. 1997. Characterization of the mucosal cell-mediated immune response in IL-2 knockout mice before and after the onset of colitis. Immunology. 91:73-80.

11. Picarella, D., et al. 1997. Monoclonal antibodies specific for beta 7 integrin and mucosal addressin cell adhesion molecule-1 (MAdCAM-1) reduce inflammation in the colon of scid mice reconstituted with CD45RBhigh CD4+ T cells. J. Immunol. 158:2099-2106.

12. Podolsky, D.K., et al. 1993. Attenuation of colitis in the cotton-top tamarin by anti-alpha 4 integrin monoclonal antibody. J. Clin. Invest. 92:372-380.

13. Hesterberg, P.E., et al. 1996. Rapid resolution of chronic colitis in the cotton-top tamarin with an antibody to a gut-homing integrin alpha 4 beta 7 . Gastroenterology. 111:1373-1380.

14. Farkas, S., et al. 2006. Blocking MAdCAM-1 in vivo reduces leukocyte extravasation and reverses chronic inflammation in experimental colitis. Int. J. Colorectal Dis. 21:71-78.

15. Kato, S., et al. 2000. Amelioration of murine experimental colitis by inhibition of mucosal addressin cell adhesion molecule-1. J. Pharmacol. Exp. Ther. 295:183-189.

16. Matsuzaki, K., et al. 2005. In vivo demonstration of $\mathrm{T}$ lymphocyte migration and amelioration of ileitis in intestinal mucosa of SAMP1/Yit mice by the inhibition of MAdCAM-1. Clin. Exp. Immunol. 140:22-31.

17. Goto, A., Arimura, Y., Shinomura, Y., Imai, K., and Hinoda, Y. 2006. Antisense therapy of MAdCAM-1 for trinitrobenzenesulfonic acid-induced murine colitis. Inflamm. Bowel Dis. 12:758-765.
18. Hamamoto, N., et al. 1999. Inhibition of dextran sulphate sodium (DSS)-induced colitis in mice by intracolonically administered antibodies against adhesion molecules (endothelial leucocyte adhesion molecule-1 [ELAM-1] or intercellular adhesion molecule-1 [ICAM-1]). Clin. Exp. Immunol. 117:462-468.

19. Sans, M., et al. 1999. VCAM-1 and ICAM-1 mediate leukocyte-endothelial cell adhesion in rat experimental colitis. Gastroenterology. 116:874-883.

20. Taniguchi, T., et al. 1998. Effects of the anti-ICAM-1 monoclonal antibody on dextran sodium sulphateinduced colitis in rats. J. Gastroenterol. Hepatol. 13:945-949.

21. Ueha, S., et al. 2007. Intervention of MAdCAM-1 or fractalkine alleviates graft-versus-host reaction associated intestinal injury while preserving graftversus-tumor effects. J. Leukoc. Biol. 81:176-185.

22. Targan, S.R., et al. 2007. Natalizumab for the treatment of active Crohn's disease: results of the ENCORE Trial. Gastroenterology. 132:1672-1683.

23. Berger, J.R., and Koralnik, I.J. 2005. Progressive multifocal leukoencephalopathy and natalizumab--unforeseen consequences. N. Engl. J. Med. 353:414-416

24. Feagan, B.G., et al. 2005. Treatment of ulcerative colitis with a humanized antibody to the alpha4beta7 integrin. N. Engl. J. Med. 352:2499-2507.

25. Powrie, F., Leach, M.W., Mauze, S., Caddle, L.B., and Coffman, R.L. 1993. Phenotypically distinct subsets of CD4+ T cells induce or protect from chronic intestinal inflammation in C. B-17 scid mice. Int. Immunol. 5:1461-1471.

26. Liu, H., Hu, B., Xu, D., and Liew, F.Y. 2003. $\mathrm{CD} 4+\mathrm{CD} 25+$ regulatory $\mathrm{T}$ cells cure murine colitis: the role of IL-10, TGF-beta, and CTLA4.J. Immunol. 171:5012-5017.

27. Strober, W., Fuss, I.J., and Blumberg, R.S. 2002. The immunology of mucosal models of inflammation. Annu. Rev. Immunol. 20:495-549.

28. Stassen, M., et al. 2004. Human CD25+ regulatory T cells: two subsets defined by the integrins alpha 4 beta 7 or alpha 4 beta 1 confer distinct suppressive properties upon CD4+ Thelper cells. Eur. J. Immunol. 34:1303-1311.

29. Denning, T.L., Kim, G., and Kronenberg, M. 2005. Cutting edge: CD4+CD25+ regulatory $\mathrm{T}$ cells impaired for intestinal homing can prevent colitis. J. Immunol. 174:7487-7491.

30. Annacker, O., et al. 2005. Essential role for CD103 in the $\mathrm{T}$ cell-mediated regulation of experimental colitis. J. Exp. Med. 202:1051-1061.

31. Braun, C., Zahn, R., Martin, K., Albert, E., and Folwaczny, C. 2001. Polymorphisms of the ICAM-1 gene are associated with inflammatory bowel disease, regardless of the p-ANCA status. Clin. Immunol. 101:357-360

32. Low, J.H., et al. 2004. Inflammatory bowel disease is linked to $19 \mathrm{p} 13$ and associated with ICAM-1. Inflamm. Bowel Dis. 10:173-181.

33. Matsuzawa, J., et al. 2003. Association between K469E allele of intercellular adhesion molecule 1 gene and inflammatory bowel disease in a Japanese population. Gut. 52:75-78.

34. Mora, J.R., et al. 2003. Selective imprinting of guthoming T cells by Peyer's patch dendritic cells. Nature. 424:88-93.

35. Barreau, F., et al. 2007. CARD15/NOD2 is required for peyer's patches homeostasis in mice. PLOS ONE. 2:e523.

36. Gullberg, E., and Soderholm, J.D. 2006. Peyer's patches and $\mathrm{M}$ cells as potential sites of the inflammatory onset in Crohn's disease. Ann. N. Y. Acad. Sci. 1072:218-232. 